\title{
Studies on Melanin.
}

VII. Melanodermatitis faciei.

By

Susumu Iijima.

(饭 島 進)

(From the Department of Dermatology, Faculty of Medicine, Tohoku University, Sendai. Director: Prof. M. I to.)

(Received for publication, April 22, 1949)

Owing to the abnormal conditions of life under the World War II, it was quite naturally expected that unusual dermatoses are to be seen. Our melanosis is a specimen in point. What we call melanosis, first appearing during the war and still prevalent, is a peculiar pigmentary dermatose which frequents mosyly among females and is seen only in exceptional instances in the male. The affected area is chiefly face and neck. The skin color, unlike that of chloasma, varies from slaty blue to dark brown, and the number of the patients amounted to 68 and is still increasing. Such pigmentary disturbance was observed in Japan not only in our clinic but also in many other clinics. The cause has thus far been unknown and only disposition plus irritation through coarse cosmetics has been vaguely suspected. We have been studying its etiology since 1941 and have finally come to a conclusion.

We do not know whether melanosis were so frequently seen in Europe except the cases of Storck, Lutz and Garnier. In America Fiesler, Spiegel, Appel, Felsher, Rothman, Curth and Andrews and other authors have reported similar melanosis.

\section{Historical Review.}

During the past World War, Riehl of the Vienna school reported a sort of melanoderma which is known as Riehl's melanosis. In the following year Hoffmann and Habermann described melanodermitis toxica lichenoides et bullosa. The great resemblance of these two dermatoses with ours as well as reported cases in Japan is accepted by all Japanese authorities. Even the similarity of the two above mentioned dermatoses, however, has since been eagarly discussed. Hoffmann tried to hold Riehl's cases to his own, while Riehl insisting upon the autonomy of his own cases.

Now let us compare Riehl's cases with Hoffmann's according to the exhaustive descriptions of $\mathrm{Kerl}^{11}$ and Habermann ${ }^{2)}$ on the German literature. 
Kerl takes it as a very rare disease, the frequency of which is only fifteen to fifty thousand. Habermann, on the contrary, emphasizes the resemblance with already known dermatoses from tar or lubricating oil. As to its etiology, Kerl excludes all possible exogenous factors while Habermann admits external origin of his cases and emphasizes fluorescent acridine derivatives as causative agents.

Kerl concludes that Riehl's melanosis is caused by a toxemia of nutritional origin, probably resulting from the intake of dopa-like substances in vicia fava. But, the fact is, neither by feeding animals with horse beens alone nor by injection of unoxidated dopa solution can man see pigment formation. Habermann attributed hyperkeratotic and follicular type to external origin and apart from these ascribed pigmentary form to photosensitive substances accumulated by inhalation.

As to clinical symptoms, Kerl could easily distinguish his melanosis from others and emphasized its gradual formation, exclusive involvement of the face and the sculp, predominance of pigmentation, the absence of inflammatory changes and the histological intactness of the epidermis. But as he must later enlarge his category, these characteristics cannot be absolute. The naming of melanosis has since been much confused. Some authors reported melanosis of apparent occupational origin under the title of Riehl's melanosis. Sharlit is of opinion that melanosis with dermal pigmentation should be summarized under the title of Riehl's melanosis.

In our cases, some resemble Riehl's cases, others with Habermann's and between them are many undistinguishable ones. In fact some show much resemblance with pellagra or pellagroid dermatoses, some look like poikiloderma or erythematodes, while others show slaty bluish pigmentation only and some show mostly hyperkeratotic follicles and comedos-all these can be arranged in series. We then take it rational to summarize all pigmentary and hyperkeratotic and comedo-forming types under the common title of Melanodermatitis faciei. Especially in one comedo-forming type I could produce pigmentation with causative cold-cream, and in one pigmentary form comedo was the first complaint and pigmentation appeared later. These facts show that they have common etiology and have only conditional differences. But here for convenience, we divide them into three cardinal types according to three main symptoms-into pigmentary, comedo-forming and keratotic types.

\section{Report of Cases.}

Age, date of onset, proximate causes and prodroms are shown in the following tables. Among 68 cases only 3 were male patient. 
TAB LE I. Age.

Pigmentary

Comedo-forming

Keratotic

Under $20 \quad 20-29 \quad 30-39 \quad 40-49$

$\begin{array}{rrrrrrr}0 & 11 & 10 & 6 & 4 & 1 & 32 \\ 1 & 20 & 5 & 4 & 1 & 0 & 31 \\ 0 & 3 & 1 & 1 & 0 & 0 & 5\end{array}$

TABLE II. Onset.

Jan. Feb. Mar. Apr. May. June. July. Aug. Sept. Okt. Nov. Dec.

Pigmentary

Comedo

Keratotic

$\begin{array}{llllllllllll}1 & 3 & 3 & 2 & 2 & 2 & 2 & 8 & 2 & 1 & 1 & 1 \\ 4 & 4 & 2 & 2 & 1 & 4 & 1 & 5 & 4 & 0 & 2 & 0 \\ 0 & 1 & 0 & 0 & 1 & 1 & 1 & 1 & 0 & 0 & 0 & 0\end{array}$

From these tables are shown that comedo-forming and keratotic types are seen most frequently in young females, while pigmentary form can occur in women over 30 years of age.

The frequent occurrence of the first type in August will be later mentioned.

Prodroms. Itching is the most frequent prodrom and is complained in each type. Erythema, and desquamation and swelling, however, are not noted in the comedo-forming and keratotic type. Noteworthy is the fact that in one case of the first type, comedo-formation was seen first and that the pigmentation appeared later on.

Proximate cause. Many substances are blamed as causative agents, of which the commonest one is cosmetics. In the pigmentary cases, sunlight and other actinic rays are also blamed.

Clinical symptoms. Brief summary of the clinical symptoms of the three types are as follows.

1. Pigmentary form. The frequent occurrence during the warmer time of the year was noted above. This helps me to assume photosensitization through coarse cosmetics. Freund saw pigmentation following the use of eau d'cologne only after irradiation by sun-light, and later perspiration was found to play a part in its etiology. The same relationship to actinic rays must be emphasized in my cases, too. Sometimes prodroms as erythema, swelling, itching and desquamation are complained. In one case such fits were the first complaint and pigmentation grew darker after each fit.

Hormonal disturbances and hypotonia, positive Aschner's reaction, as are often observed, indicate the instability of endocrine and autonomy nervous system. In most of them iodic acid value of the blood serum is markedly enhanced.

As regarding to the localisation, temporal, preauricular and mental region never fail to show pigmentation. Other parts of the face, retroauricular portion and the sides of the neck are also usually involved. In 
three cases "butterfly-form" of the skin lesion was noted. The skin-colour varies from light slaty blue to dark violet, but unlike that of chloasma, tone of violet is always ascertainable. The skin surface in some cases is as coares as a grater, in others rather smooth and looks like powdered. Sometimes flat elevations of the skin fields are seen, but typicallichen planus-like papule is found by none. Erythema is noticed only in initial cases. By first glance, tanning forms rather diffuse patches, but on closer observation is found either in reticulated arrangement corresponding to the superficial network of blood vessels, or situated perifollicular. Direct surroundings of the follicular openings are rather free from tanning. Atrophy was not markable and was seen only in exceptional cases. In three cases the sculp was involved, in one of which tinea amiantacea-like enclosement of the hair was found.

Pigment macules lose their intensity at retroauricular region and diminish into tiny follicular patches. Finally they extinguish on the borderline to breast. Other parts of the body are usually free from tanning, except in one who carried pigment patches on the flexor surface of forearms. In some cases, pigmentation on the conjunctiva and on the buccal mucous membrane was noted, but yellow disclolration of the sclera was not seen. Amongst the 32 cases of pigmentary type are three intermediate ones which in addition to slight smutty pigmentation on the temporary region, showed follicular keratotic pluggings and comedos. In these three cases perifollicular infiltration was noted histologically. And pigment cells were seen chiefly in the follicular vicinity. Tendency to seborrhea was complained by all.

2. Comedo form. Forehead is rather slightly affected. Outer part of the eyebrows, zygomatic region, sides of the cheeks and mandibular angles are closely laden with a band of follicular papules, pustules and especially with black or skin-colored horny pluggings. Pigmentation, if any, is not prominent and is locallized mostly near follicular openings. Pigment cells are not found microscopically.

3. Keratotic form. Both sides of the cheeks, preauricular region are chiefly affected. Even the nape of the neck was affected in one. Each papule is almost as large as rice grain, follicular located and is usually skincolored but sometimes livid violet, abnormally hard and renders the skin the feeling of a grater. In typical cases almost similar exanthemas are seen, but in some pustules are mixed.

This type visited us successively in summer of 1945, and were successfully treated with cod's liver oil or butter intake. This suggests us that this type had occured upon the deficiency of vitamin A and D. The dark adaptation of these cases, however, was not examined because they failed to visit us till several weeks later, when the skin was entirely clear of erup- 
tion. Although vitamin $\mathrm{A}$ and $\mathrm{D}$ are reported to be effective in acne vulgaris, we could not get successful results in comedo forms as in this.

Histopathology and histochemical findings. In eight cases biopsy specimens were excised. They are obtained from six pigmentary cases containing one intermediate case and two comedo-forming cases.

Specimen No. 1. Section obtained from a female patient of 31 years of age complaining pigmentation of the face for two months. Epidermis is thinner than usual with shorter processus. Basal layer is laden with little pigment. In the papillary and subpapillary region, espec. near the hair follicles, are situated pigment cells close to blood vessels. Melanin granules are seen also in reticulum cells. Inflammatory changes have utter subsided.

Specimen No. 2. Obtained from a 30 years old woman. Duration of the pigmentation more than five years. Thin epidermis. No hyperkeratosis. Some intraepidermal vacuoles are found. Perivascular infiltrate in the upper dermal layer consists of lymphocytes and hictiocytic cells. Chromatophores are increased in number. Melanin granules are phagocyted in the reticulum cells, too. Elastic fiber is lessened in the infiltrated parts.

Specimen No. 3. Section obtained from a 37 years old female patient with the pigmentation of the face of three years duration. Epidermis is slightly parakeratotic. Rete thickened. Basal layer is poor in pigment. In the corium perivascular edema and infiltrats of round and histiocytic cells are noticed. These reach to the middle layer of the corium. Pigment cells are distributed unevently in the papillary and subpapillary layer.

Specimen No. 4. This section was excised from a 29 years old female patient with severe dark brown pigmentation of the face of two months duration. Epidermis is somewhat disordered and vacuolated near follicular openings. The basal layer shows subnormal pigment content. Hair follicles strongly dilated and filled with horny masses. In the corium are seen moderate perivascular and perifollicular infiltrates cosisting of round and histiocytic cells in the subpapillary layer, where numerous melanophores are found especially at the vascular surroundings.

Specimen No. 5. Section obtained from a 28 years old female patient. Duration of pigmentary disturbance 4 months. Rete cells vacuolated and loosely connected. Borderline to the corium not distinct. Dilation of follicular openings are remarkable. Here and there intraepidermal cellular infiltrates are noticed. Basal layer contains subnormal amount of melanin granules. Numerous pigment cells are situated in the papillary layer. In the deeper layer of the derma, round and histiocytic cells and chromatophores are seen in the vicinty of blood vessels.

Section No. 6. was excised from a 54 years old female with pigmentation of one years duration. Pigment content of the basal layer is some- 
what increased. Follicular openings are strongly dilated, in which fat and horny masses are found. In the corium are seen hyperemia in the upper layer and round cell infiltration in the surroundings of blood vessels, where pigment is situated in melanophores and in connective tissue fibers. Marked edema and cellular infiltration are seen in the follicular vicinity.

Specimen No. 7. Excised from a 30 years old woman complaining itching and erythema and comedo formation on both cheeks for about three months. Epidermis is thinner than usual and no epidermal processus are seen. Basal layer contains normal amount of pigment. No pigment cells in the derma. In the subpapillary layer blood vessel are dilated and edematous. Hair follicles are expanded, contain comedos. The basal layer of hair follicles is rich in pigment. Degeneration of arrector muscle is noted.

Specimen No: 8 was obtained from a 22 years old female patient, who after the use of a cold-cream for a short time, noticed numerous comedos and pustules on both cheeks. Miscroscopically epidermis is flattened at follicular openings. Basal layer, which is rich in melanin, is somewhat disordered. Apparent hyperemia in the upper dermal layer is noted. Cystic enlargement of hair follicles is seen even macroscopically. The tissues surrounding them are pressed aside, but show little or no inflammatory changes.

Brief summary of the histo-pathological findings is as followes.

In the first type,the epidermis shows various stages of degenerative changes according to the clinical signes of acuity. Common characteristics lies in the diminished or no pigment of the basal layer and in the presence of various numbers of pigment cells in the upper dermal layer. In most of the cases perivascular infiltrates consisting of round and histiocytic cells are seen. The affection of the follicle apparatus differs by cases, but, generally speaking, grows severer as the clinical appearance of comedo takes place.

In the comedo-forming type the main aspect lies in the utmost dilatation of hair follicles.

In all these specimens are noted four cardinal pathological features-degeneration of epidermis, affection of hair follicles, increase of pigment cells in the corium and inflammatory changes in the corium. And it is made clear that they show gradual passing from one type to another.

In the specimen No. 3, I stained the section with dopa and got, unexpectedly, positive results. The author tried the dopa-reaction after the variation of Fujiwara, ${ }^{3)}$ and found that after 90 minutes in $37^{\circ} \mathrm{C}$. small pigment cells of the upper dermal layer undoubtedly darkened. As the free melanin granules in that layer are not stained black, it is obvious that there is no absorption of oxydated dioxyphenylalanin by melanin granules. 
The dopa-reaction of Bloch ${ }^{4}$ has been estimated to be an indicator of the pigment forming facility of a cell, which has since been attributed only to the dendritic cells of the basal layer and to dermal melanoblasts in certain cases. But the true nature of this reaction is still under discussion. Especially the discussions of Meirowsky and Katsunuma are not throughly answered. So, in order to confirm our results, we further stained two sections with peroxydese-reagence and with Unna's oxygen-space-method. The results were quite corresponding-that is, by both Unna's and Okano's ${ }^{51}$ methode dermal pigment cells were found to contain peroxydase granules, and that the less the melanin content, the more the peroxydase just as Watanabe ${ }^{6}$ ) in our clinic proved in several pigment producing tissues. And by Unna's axygen-space-method, infiltrated parts of dermal layer is stained deep green as oxygen space and especially strongly stained is the vicinity of blood vessels-in short, where the pigment cells are located, there is ample amount of free oxygen.

\section{Epiology and Pathogenesis.}

With regards to the etiology of this dermatose, we will take up photosensitisation through coarse cosmetics as external factor and hypovitaminosis and hormonal disturbances as internal, and as a supplementary factor dysharmony of the autonomy nervous system.

Hoffmann and Habermann ascribed hyperkeratotic form and folliculitis in his cases to external contact with petroleum and pitch, and apart from these, contributed pigmentary form-melanodermitis in the sence of the word-to inhalation of toxic substances. Riehl and Kerl attached great importance to the nutritional origin of their cases and excluded all possible external irritants. Most of our cases are women of indoor activities having no occupational contact with tar, pitch or lubricating oil. Professional causes can be distinctly excluded. Neither can nutrition alone explain such occurrance. So it is quite natural that most Japanese authors took up crude cosmetics as causative agents. Shortly after the War, Tanimura and Hayami reported acne-like eruption from the use of cold-cream. But as to the irritants in it, the former finds cause in insaturated fatty acids, while the latter in lower hydrocarbons. Recently Kitamura") blamed fluorescent substance contained in oil used for cooking as a cause of melanosis. We analysed cosmetics obtained from patients. All have flavour of bergamotte oil, and in three of them lower hydrocarbons were found to be the chief component. These bergamotte oil and lower hydrocarbons we take up as external causative agents.

It is well admitted that spread of fat or oil on the skin increases pigmentation by irradiation. Nakajo ${ }^{8)}$ in our clinic showed the inhibitory action of vitamin $\mathrm{C}$ and $\mathrm{B}_{2}$ against the pigmentation by ultraviolet irradia- 
tion after tar or bergamotte oil spread. According to his theory, this action is due to the decreased permeability of the skin by vitamin $C$ and $B_{2}$. So it is quite naturally expected that in $\mathrm{B}_{2}$ or $\mathrm{C}$ - hypovitaminotic individuals the skin is much more susceptible to external irritants (in this case, to bergamotte oil or lower hydrocarbons) than normal persons. In fact, the vitamin $\mathrm{C}$ content of the patients' skin measured by 2,6 , dichlorphenolindophenolmethod is distinctly lower than normal controls. From this is clear that the hypovitaminitic stage induced by the deficient supply of nourishment during the war, enhanced the susceptibility of the skin against various photosensitizing substances.

On the other hand, vitamin $\mathrm{C}$ exerts inhibitory action on the pigment formation as shown by the supressive effects to dopa-reaction and to the peroxydase reaction. That is, vitamin $\mathrm{G}$ deficiency contributes to the etiology of this disease in duplicated way-first by enhancing the penetrability of the skin, secondly by the loss of inhibitory action against pigment production. Cutaneous manifestation of vitamin $\mathrm{C}$ deficiency is known as scurvey, but its former stage, together with anorexia, arthralgia and tachycardia, shows follicular hyperkeratosis and pigmentation. But as general complaints were rare in our cases, ours is not true hypovitaminosis or dysvitaminosis, but a sort of paravitaminosis in Comel's ${ }^{9)}$ sence.

As to vitamin A and D, Kitamura obtained no definitive results in dark adaptation in his cases. Hypovitaminosisacne of American authors ${ }^{10}$ may have resemblance, but general dryness of the skin was not observed in our cases except in one who carried slight follicular keratosis on the extensor surface of extremities. However, the successful treatment with cod's liver oil shows the participation of these vitamins at least in pure keratotic type.

In the former parts we admitted possible photosensitization through bergamotte oil or lower hydrocarbons or acridine derivatives. But, here too, the participation of another vitamin cannot be overlooked. Ito ${ }^{11}$. could cure pellagra with intake of vitamin B-complex and later with vitamin $B_{1}$ alone. Later vitamin $B$-complex deficiency was found to cause pellagra or pellagroid dermatose. In some of our cases, the lesions were localized on both cheeks in butterfly-form accompanied with other clinical signs of vitamin B-complex deficiency. Hasebroek also reported similar case in his melanosis. Such facts suggest to us the participation of vitamin B-complex through successful treatment with this alone was inprocurable. In short vitamin B deficiency must have taken part in this disease accompanied with the enhancement of the permeability of the skin.

As to the relationship of autonomy nervous system with the pigment formation, Ito has already made exhaustive researches which make it unnecessary to touch here again. 
The role of adrenals and ovaries in case of melanosis was noted by Comel and Rolba. The irregularity of menstrual cycles was usually complained in pigmentary cases. Recently Suzue and Yamamoto ${ }^{12}$ reported a case of vagabond's disease with extensive degeneration of adrenals. Some of our cases showed hypotonia and pigmentation on the buccal mucous menbrane and were successfully treated with prolonged injection of the cortical horman of adrenals. From these facts the role of the endocrine system cannot be overlooked.

Pathogenesis. The point of discussion between Bloch's school and Meirowsky ${ }^{13)}$ lies in the pigment forming facilities of superficial pigment cells in the corium in man. These pigment cells increase much in number in cases of melanosis and form the greatest characteristics. In all reported histopathology of melanosis, these cells are dopanegative and are attributed to no melanogenic activities, except that of Shwartz's case who admitted "primary activity in the CHROMATOPHORES."

Meirowsky, though he admits the negative dopa-reaction of the corium cells, persists on the mesodermal origin of melanin, supported by Lipschütz's animal experiments. Judging from our histologic and histochemical findings, the pigment content of the derma never goes parallel with that of the epidermis. The abundant store of melanin by almost intact epidermis cannot be explained by Bloch's theory only. Besides, the assumption of the loss of pigment retaining facility of epidermal cells is too hypothetic.

From above mentioned researches the author concludes as follows: Owing to the enhanced permeability of the skin induced by vitamin $\mathrm{C}$ and B-complex deficiency, the component of crude cosmetics, lower hydrocarbons reaches corium. Where with the aid of vitamin B-complex deficiency it causes photosensitization and, as the results, abnormal oxygenspace is prodused in derma. But in some cases, where perivascular region is especially rich in oxygen, the hematogenous distribution of photocatalytic substances cannot be excluded.

Oizumu ${ }^{14}$ ) found higher content of iodic acid reducing substances in blood serum in cases of pigmentary form. This suggests the possibility that both premelanin-tryptophane or tyrosine-and catalyser reach derma through blood vessels. And the positive peroxydase reaction. Though peroxydase does not always coincides with oxygen-space, oxygen-space usually contains peroxydase. Thus the oxidizing potency is extremely enhanced and then the pigment formation takes place in the presence of percxydase. Whether the peroxydase concerned is of true fermentative nature or only pseudoperoxydase, it does not matter. And where the oxidizing potency is high and pigment production takes place, positive dopa-reaction is naturally expected. Thus the more rational dermal pigment formation in our melanosis is explained. 
In cases where follicular eruptions are predominant, the inva le of cosmetics takes place chiefly through pilous poles, and cause acnelike eruption or, supported by the deficiency of vitamin A, cause hyperkeratotic papules. Even in such cases pigment formation may occur as shown in the intermediate cases.

\section{Summary and Conclusions.}

After the historical review of melanosis, 68 cases of melanodermatitis observed in Tohoku University were reported.

From the clinical and histological observations they were divided into three types. The characteristics of each type is discribed. All these types have a common etiology and have only conditional differences.

As to the etiology, the role of subclinical vitamin deficiencies and irritative cosmetics was emphasized. In one case dermal pigment formation was certified with positive dopa- and peroxydase-reaction.

\section{References.}

1) Kerl, Arch. Derm. u. Syph., 1921, 130, 436.

2) Habermann, Derm. Zschr., 1920, 30, 63.

3) Fujiwara, Okayama med. J., 1929, 41, 474.

4) Bloch, Jadassohns Handb. I, 1927.

5) Okano, Jap. J. Microbiol., 1926-27, 20-21, 1149, 1305.

6) Watanabe, Tohoku med. J., 1947, 1-2, 1. (abstracted in Tohoku J. of Exp. Med., 1949, 50, 384.)

7) Kitamura, Tokyo med. J., 1928, 65, 14.

8) Nakajo, Jap. J. Derma. \& Ven., 1942, 52, 132. (abstracted in Tohoku J. of Exp. Med., 1949, 50, 354.)

9) Comel (cited by Goodman), Arch. Derma. \& Syph., 1938, 38, 389.

10) Loewenthal, Arch. Derma. \& Syph., 1933, 28, 700.

11) Ito. Jap. J. Derma. \& Urol., 1923, 24, 240.

12) Suzue and Yamamoto, Medicine, 1948, 5, 17.

13) Meirowsky, Zbl. Haut- u. Ges. Krankh., 1923, 8, 97.

14) Oizami, Acta dermatol. jap., 1949, 45, 37. 